\title{
Increase in HDAC9 suppresses myoblast differentiation via epigenetic regulation of autophagy in hypoxia
}

Zhang Zhang ${ }^{1}$, Liqiang Zhang ${ }^{2}$, You Zhou ${ }^{3}$, Liya Li ${ }^{2}$, Jiangdong Zhao ${ }^{4}$, Wen Qin², Zuolin Jin $^{5}$ and Wenjia Liu ${ }^{2}$

\begin{abstract}
Extremely reduced oxygen $\left(\mathrm{O}_{2}\right)$ levels are detrimental to myogenic differentiation and multinucleated myotube formation, and chronic exposure to high-altitude hypoxia has been reported to be an important factor in skeletal muscle atrophy. However, how chronic hypoxia causes muscle dysfunction remains unknown. In the present study, we found that severe hypoxia $\left(1 \% \mathrm{O}_{2}\right)$ significantly inhibited the function of $\mathrm{C} 2 \mathrm{C} 12$ cells (from a myoblast cell line). Importantly, the impairment was continuously manifested even during culture under normoxic conditions for several passages. Mechanistically, we revealed that histone deacetylases 9 (HDAC9), a member of the histone deacetylase family, was significantly increased in C2C12 cells under hypoxic conditions, thereby inhibiting intracellular autophagy levels by directly binding to the promoter regions of Atg7, Beclin1, and LC3. This phenomenon resulted in the sequential dephosphorylation of GSK3 $\beta$ and inactivation of the canonical Wnt pathway, impairing the function of the $\mathrm{C} 2 \mathrm{C} 12$ cells. Taken together, our results suggest that hypoxia-induced myoblast dysfunction is due to aberrant epigenetic regulation of autophagy, and our experimental evidence reveals the possible molecular pathogenesis responsible for some muscle diseases caused by chronic hypoxia and suggests a potential therapeutic option.
\end{abstract}

\section{Introduction}

Chronic high-altitude hypoxia contributes to the muscle atrophy observed in patients with pathologies associated with a hypoxic microenvironment, such as chronic obstructive pulmonary disease (COPD) and arteriosclerosis obliterans ${ }^{1}$. Some studies have reported that the hypoxia-induced inhibitory effect on muscle regeneration is a temporary and reversible process ${ }^{2}$, which seems to delay myogenic differentiation. By contrast, some studies have suggested that impaired regeneration under chronic

\footnotetext{
Correspondence: Zuolin Jin (zuolinj@163.com)Wenjia Liu (wenjia@xiterm.com) (wenjialiu23@163.com)

${ }^{1}$ General Surgery Department, Tang Du Hospital, Fourth Military Medical University, 710032 Xi'an, Shaanxi, China

${ }^{2}$ State Key Laboratory of Military Stomatology \& National Clinical Research Center for Oral Diseases \& Shaanxi International Joint Research Center for Oral Diseases, Center for Tissue Engineering, School of Stomatology, Fourth Military Medical University, 710032 Xi'an, Shaanxi, China

Full list of author information is available at the end of the article.

These authors contributed equally: Zhang Zhang, Liqiang Zhang, You Zhou Edited by GM Fimia
}

hypoxia has long-lasting effects that may not be sufficiently reversed and results in muscle mass loss ${ }^{3,4}$; however, the underlying mechanism remains unclear.

Myogenesis is an essential step for muscle regeneration. In addition, myoblasts are required for this process because, by successfully differentiating and fusing with each other, they regenerate the characteristic multinucleated myofibers; they also play an important role in maintaining muscle structure and mass ${ }^{5}$. Some studies have shown that $1 \% \mathrm{O}_{2}$ represses the myogenic differentiation of $\mathrm{C} 2 \mathrm{C} 12$ (from a myoblast cell line) ${ }^{2,6}$, whereas a $3-6 \% \mathrm{O}_{2}$ level can promote myogenesis ${ }^{7}$. Previous studies have reported that severe hypoxia negatively regulates myogenic differentiation by inhibiting MyoD or Myogenin in myoblasts in a manner that is dependent on ${ }^{3}$ or independent of ${ }^{6,8}$ hypoxia-inducible factor (HIF1 $\alpha$ ), a major contributor to the response to hypoxia signaling. Some studies have noted that myoblast differentiation is a reversible injury caused by hypoxia because myoblasts

\section{(c) The Author(s) 2019}

\footnotetext{
(c) (i) Open Access This article is licensed under a Creative Commons Attribution 4.0 International License, which permits use, sharing, adaptation, distribution and reproduction cc) in any medium or format, as long as you give appropriate credit to the original author(s) and the source, provide a link to the Creative Commons license, and indicate if changes were made. The images or other third party material in this article are included in the article's Creative Commons license, unless indicated otherwise in a credit line to the material. If material is not included in the article's Creative Commons license and your intended use is not permitted by statutory regulation or exceeds the permitted use, you will need to obtain permission directly from the copyright holder. To view a copy of this license, visit http://creativecommons.org/licenses/by/4.0/.
} 
retain their capacity to proliferate or differentiate when normal oxygen levels are restored ${ }^{2}$. By contrast, other researchers believe that the effect of chronic hypoxia on muscle is perennial ${ }^{3,4}$. Furthermore, our laboratory previously confirmed that microenvironment-induced functional impairments can be transmitted to daughter cells in an epigenetically regulated manner ${ }^{9}$. However, the effects of chronic hypoxia on the functional alterations of myoblasts and the intrinsic underlying mechanism of its effects have been largely unexplored.

Acetylation of lysine residues on histones is a key process of the epigenetic regulation of DNA transcription ${ }^{10}$. The acetylation levels of lysine residues on histones are controlled by lysine acetyltransferases (KATs)/histone acetyltransferases (HATs) and histone deacetylases (HDACs). In the past several years, some studies have shown that HDACs respond to long-term hypoxiainduced gene transcriptional regulation ${ }^{11}$, adipocyte dysfunction $^{12}$, and disease development ${ }^{13,14}$. Importantly, it has been demonstrated that histone deacetylase is involved in muscle-specific genes and in the regulation of muscle differentiation ${ }^{15,16}$. Therefore, we hypothesized that hypoxia might induce persistent changes in myoblasts via epigenetic regulation that causes subsequent muscle dysfunction.

In this study, we demonstrated that a high-altitude hypoxic microenvironment impaired the function of $\mathrm{C} 2 \mathrm{C} 12$ in an epigenetically regulated manner. Mechanistically, we revealed that HDAC9 was significantly increased in the $\mathrm{C} 2 \mathrm{C} 12$ due to hypoxia, thereby inhibiting intracellular autophagy by binding directly to the promoter regions of Atg7, Beclin1, and LC3. Decreased autophagy resulted in dephosphorylation of GSK3 $\beta$ and subsequent inactivation of the canonical Wnt pathway, impairing the myogenesis in the $\mathrm{C} 2 \mathrm{C} 12$.

\section{Results}

\section{Hypoxia inhibits the properties of $\mathrm{C} 2 \mathrm{C} 12$ cells}

To investigate the properties of the $\mathrm{C} 2 \mathrm{C} 12$, myogenesis induction was performed. The $\mathrm{C} 2 \mathrm{C} 12$ successfully formed myotubes and showed elevated expression of MyoG and MyoD (Supplementary Fig. 1), as indicated by qRT-PCR and western blotting. To investigate the effect of hypoxia on the function of myoblasts, the $\mathrm{C} 2 \mathrm{C} 12$ cells were exposed to hypoxia $\left(1 \% \mathrm{O}_{2}\right)$ or were maintained under standard conditions $\left(21 \% \mathrm{O}_{2}\right)$. The morphology of the $\mathrm{C} 2 \mathrm{C} 12$ was mostly unaltered under the hypoxic microenvironment; however, the number of granules was increased in the cytoplasm, and the nucleus was obviously visible (Fig. 1a). The viability and proliferation of the $\mathrm{C} 2 \mathrm{C} 12$ were significantly reduced in the hypoxia group compared with the control groups, as shown by MTT and Edu analyses (Fig. 1b, c), indicating that although hypoxia promoted the viability of the $\mathrm{C} 2 \mathrm{C} 12$ initially, the proliferation capacity was ultimately reduced after 7 days of exposure to hypoxia. Moreover, the number of apoptotic $\mathrm{C} 2 \mathrm{C} 12$ was increased in the hypoxia group, as determined by flow cytometry (Fig. 1d).

We next examined the myogenesis of the $\mathrm{C} 2 \mathrm{C} 12$ under normoxic and hypoxic conditions. The $\mathrm{C} 2 \mathrm{C} 12$ cultured under hypoxic conditions for 7 days manifested severe inhibition of myogenesis, as indicated by reduced immunostaining and decreased expression of MyoG and MyoD (Fig. 1e, f), a finding consistent with previous reports ${ }^{2,17}$. Collectively, our results indicate that extremely low $\mathrm{O}_{2}$ levels inhibit the proliferation and differentiation capabilities of the $\mathrm{C} 2 \mathrm{C} 12$ but promote apoptosis.

\section{Hypoxia inhibits the myogenesis in $\mathrm{C} 2 \mathrm{C} 12$ cells via HDAC9}

Previous research has confirmed that the hypoxiamediated inhibition of myogenic differentiation is reversible $^{2}$. However, we observed lower expression levels of MyoG and MyoD in the $\mathrm{C} 2 \mathrm{C} 12$ of passages 2, 4, and 6 under hypoxia compared with the same passages of $\mathrm{C} 2 \mathrm{C} 12$ under normoxia (C2C12 cultured under hypoxic and normoxic conditions for 3 days were used as passage 1; Fig. 2a). The expression levels of MyoG and MyoD were almost the same with passaging, and they were lower than in the normal cells (Fig. 2b), indicating that the hypoxiamediated inhibition of myogenesis in the $\mathrm{C} 2 \mathrm{C} 12$ was consistent even when the cells were cultured under normoxic conditions for several passages.

Growing evidence suggests that the microenvironment changes the posttranscriptional modification of histones via acetylation ${ }^{18-20}$, which subsequently influences the function of cells ${ }^{12}$. Consequently, we compared the expression patterns of $\mathrm{HDAC}$ families between the $\mathrm{C} 2 \mathrm{C} 12$ cultured under normoxic conditions and the $\mathrm{C} 2 \mathrm{C} 12 \mathrm{cul}-$ tured under hypoxic conditions. The results showed that the expression levels of HDAC1, 2, 8, and 9 were continually elevated after exposure to hypoxia for $1 \mathrm{~h}, 24 \mathrm{~h}$, and 7 days. In particular, the expression of HDAC9 increased nearly 5 -fold in the hypoxia-exposed $\mathrm{C} 2 \mathrm{C} 12$ compared with the normal control cells (Fig. 2c, Supplementary Fig. 2). Next, we focused on HDAC9 and examined the expression levels of HDAC9 at different time points under hypoxia. Western blotting analysis showed that the level of HDAC9 significantly increased under hypoxia, and this effect was also apparent in different passages (Fig. 2d, f). As hypoxia usually affects cell properties via hypoxia-inducible factors (HIFs) ${ }^{3,21}$, we also examined the expression levels of HIF1 $\alpha$ and HIF $2 \alpha$ at different time points in the cells under hypoxia. Western blotting analysis showed that HIF1 $\alpha$ increased slightly after $1 \mathrm{~h}$ of hypoxia but then decreased continuously and almost disappeared after $72 \mathrm{~h}$ of hypoxia, and the expression pattern of HIF $2 \alpha$ showed no correlation with the different time points of hypoxia (Supplementary Fig. 3). 


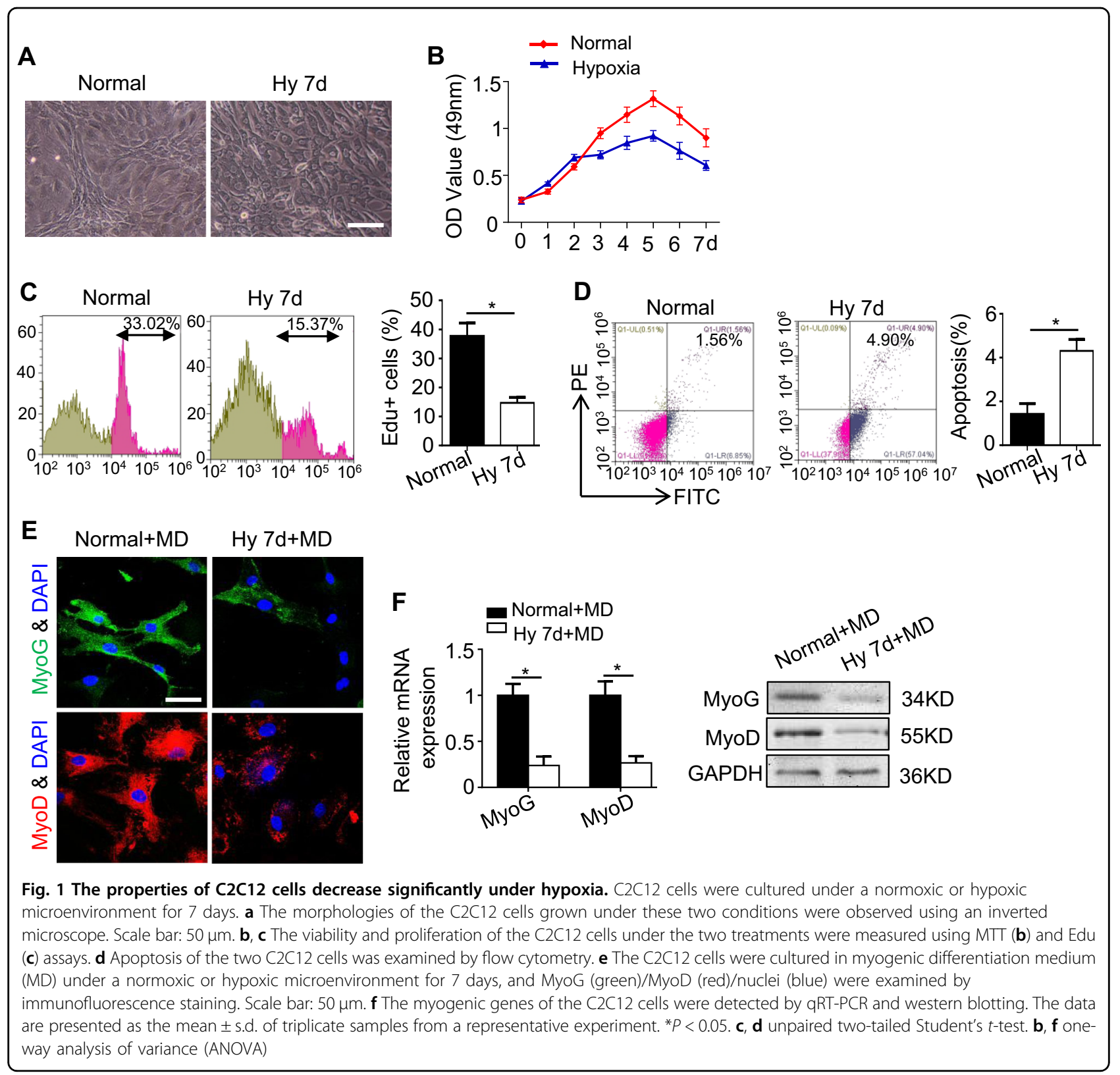

These results indicate that increased HDAC9 in the C2C12 has no close connection with HIFs; therefore, we did not study these factors in the other experiments.

HDAC9 has been found to prefer lysine 9, lysine 14, and lysine 18 of histone $\mathrm{H}^{22}$, which it acetylates to regulate gene function; therefore, we examined the acetylation sites of histones 3 and 4 after blocking the expression of HDAC9. The results showed that, compared to trichostatin A (TSA), sodium butyrate $(\mathrm{NaB})$ had a strong effect on the simultaneous inhibition of HDAC9 expression and promotion of H3K9 acetylation (Supplementary Fig. 4). Lysine residue H3K9 showed the opposite expression pattern to upregulated HDAC9. Furthermore, H3K9 was also hypoacetylated (more than 2-fold) in different passages of the hypoxia-C2C12, despite the enhanced level of HDAC9 (Fig. 2e-g), suggesting that hypoxia-induced HDAC9 elevation leads to histone deacetylation at lysine residue 9 of histone $\mathrm{H} 3$.

\section{Inhibition of HDAC9 rescues the hypoxia-induced impairment of myogenesis}

The overexpression of HDAC9 in the $\mathrm{C} 2 \mathrm{C} 12$ cultured under normoxic conditions significantly impaired their myogenesis. In contrast, the inhibition of HDAC9 expression in the $\mathrm{C} 2 \mathrm{C} 12$ cultured under normoxic conditions partially enhanced their myogenesis, as confirmed 

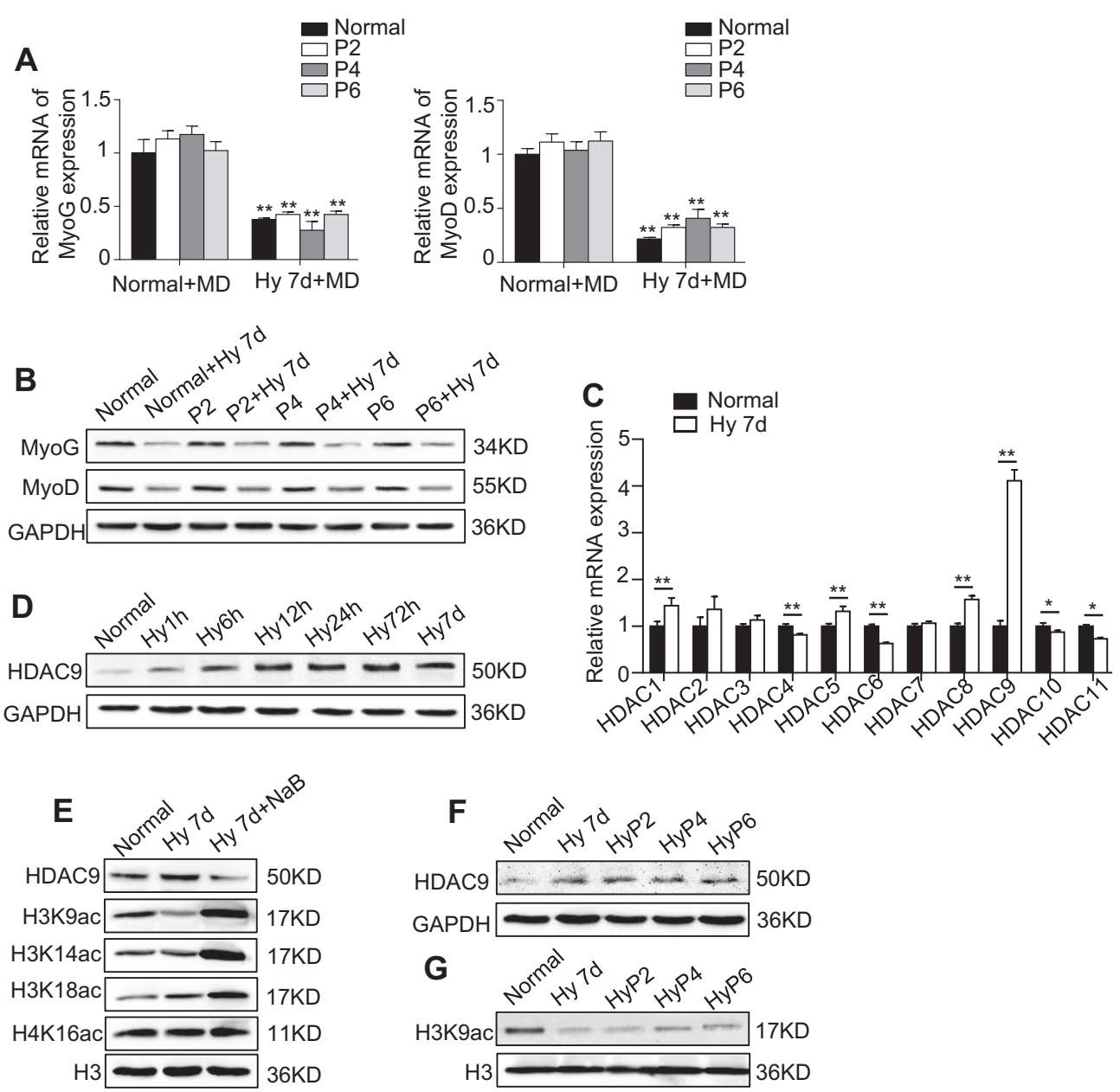

Fig. 2 The effect of hypoxia on the disrupted function of $\mathrm{C} 2 \mathrm{C} 12$ cells occurs in an epigenetic-dependent manner. $\mathbf{a}, \mathbf{b} \mathrm{C} 2 \mathrm{C} 12$ cells were cultured in MD under normoxic or hypoxic conditions for 7 days as Normal (P1) or Hypoxia P1 (Hy), respectively, and then, both were continuously passaged six times under normoxic conditions. The expression of MyoG and MyoD in passages 2, 4, and 6 in the C2C12 cells was analyzed by qRTPCR (a) and western blotting (b). c The C2C12 cells were cultured under normoxic or hypoxic conditions for 7 days, and the expression of the histone deacetylase family (HDAC1-11) was examined by qRT-PCR. $\mathbf{d}$ The expression levels of HDAC9 in the C2C12 cells at different time points after exposure to hypoxic stimuli were examined by western blotting. e The expression levels of HDAC9 and well-known histone targets of HDAC9 in normoxia or hypoxia with or without $\mathrm{NaB}$ treatment (the dose of $\mathrm{NaB}$ is $200 \mu \mathrm{M}$ ) in the C2C12 cells are shown. $\mathbf{f}, \mathbf{g}$ The expression levels of HDAC9 and H3K9ac in different passages (normal, Hy7d, HyP2, HyP4, and HyP6) of the C2C12 cells were examined by western blotting. The data are presented as the mean \pm s.d. of triplicate samples from a representative experiment. ${ }^{*} P<0.05,{ }^{* *} P<0.01$. One-way analysis of variance (ANOVA)

by MyoG and MyoD expression (Supplementary Fig. 5, Fig. 3a). These data indicate that HDAC9 regulates the myogenesis in $\mathrm{C} 2 \mathrm{C} 12$. We next investigated the therapeutic effect of downregulating HDAC9 on the functional recovery of the $\mathrm{C} 2 \mathrm{C} 12$ cultured in hypoxia. We observed that the decreased expression levels of MyoG and MyoD induced by hypoxia were successfully rescued by $\mathrm{NaB}$, suggesting that the therapeutic effect may be caused by the inhibited expression of the HDAC family that was induced by hypoxia (Fig. 3b).

Given that HDAC9 directly regulated the myogenesis in the $\mathrm{C} 2 \mathrm{C} 12$ and that $\mathrm{NaB}$ is a broad-spectrum HDAC inhibitor, we next specifically downregulated the HDAC9 level in the $\mathrm{C} 2 \mathrm{C} 12$ cultured in hypoxia. As expected, the decreased expression levels of MyoG and MyoD induced by hypoxia were also rescued after specifically downregulating HDAC9 (Fig. 3c). Collectively, these data indicate that the inhibited myogenesis of the $\mathrm{C} 2 \mathrm{C} 12$ induced by hypoxia was likely due to the elevated HDAC 9 level, and the downregulation of HDAC9 successfully rescued the $\mathrm{C} 2 \mathrm{C} 12$ impaired by hypoxia.

\section{HDAC9 inhibits myogenesis via the epigenetic regulation of autophagy}

As autophagy is required to maintain cellular function and homeostasis under hypoxic conditions ${ }^{23-25}$, we next 


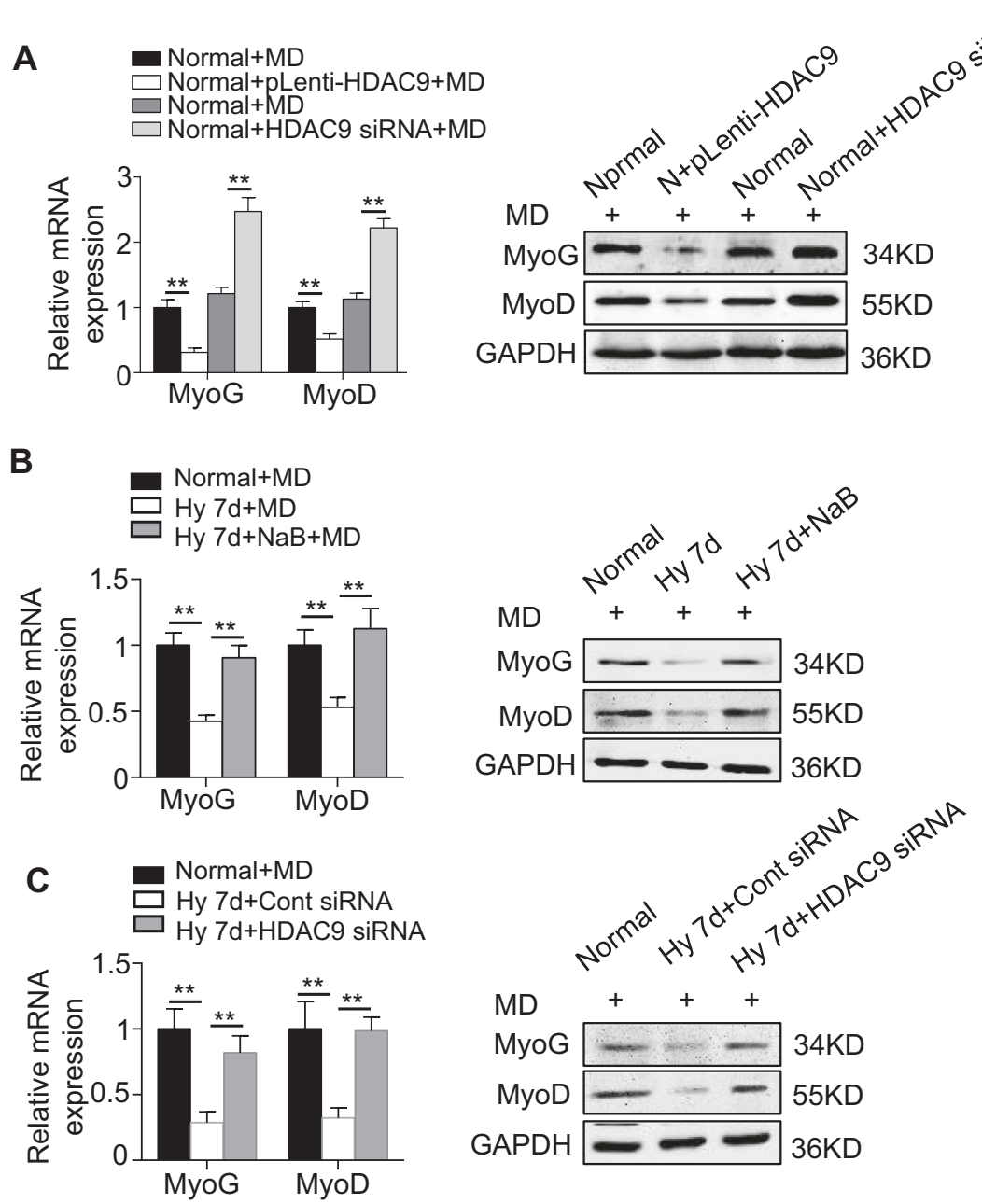

Fig. 3 Downregulation of the expression of HDAC9 by an inhibitor or siRNA rescues the inhibitory effect of hypoxia on C2C12 cell myogenesis. a The regulation of HDAC9 expression through lentiviral vectors in the $\mathrm{C} 2 \mathrm{C} 12$ cells is depicted. The expression of MyoG and MyoD was examined by qRT-PCR and western blotting at day 7 after myogenic induction. b, c To observe the effect of HDAC9 on the myogenesis in the C2C12 cells, C2C12 cells were treated with the HDAC inhibitor sodium butyrate (NaB) $(\mathbf{b})$ or with HDAC9 siRNA (c). After myogenic induction for 7 days, the expression levels of MyoG and MyoD in the C2C12 cells were examined by qRT-PCR and western blotting. The data are presented as the mean \pm s.d. of triplicate samples from a representative experiment. ${ }^{*} P<0.05,{ }^{* *} P<0.01$. One-way analysis of variance (ANOVA)

investigated the effect of hypoxia on autophagy in $\mathrm{C} 2 \mathrm{C} 12$. The expression of LC3II was found in normal cells, and this expression was decreased after exposure to hypoxia for 7 days. In contrast, the expression of p62, which acts as specific cargo degraded by autophagy, was distinctly increased under the above hypoxic conditions compared with the control group (Fig. 4a). We next applied the autophagy-flux inhibitor chloroquine (CQ), which prevents lysosome degradation, thus increasing LC3II expression significantly when autophagy is active ${ }^{26}$. The CQ experiments showed that, in contrast to the cells cultured in normoxia, the cells cultured in hypoxia lacked the capacity for further autophagosome formation, as indicated by western blotting and immunostaining (Fig. 4a, b). Furthermore, we found fewer autophagosomes in the hypoxic cell group than in the normoxic cell group, as indicated by transmission electron microscopy (Fig. 4c). These results suggest that the autophagic activity was inhibited after long-term exposure of the cells to hypoxia. Moreover, the decline in the autophagic activity persisted from P2 to P6, showing the opposite effect of HDAC9. Accordingly, a lack of autophagy led to the accumulation of p62 in the passage 2 cells cultured under hypoxia but remained constant between P2 and P6 (Fig. 4d, Supplementary Fig. 6). Importantly, autophagy in the normal $\mathrm{C} 2 \mathrm{C} 12$ was significantly activated and caused a decrease in p62 accumulation after the $\mathrm{NaB}$ treatment that downregulated HDAC9 in the presence or absence of $\mathrm{CQ}$ 

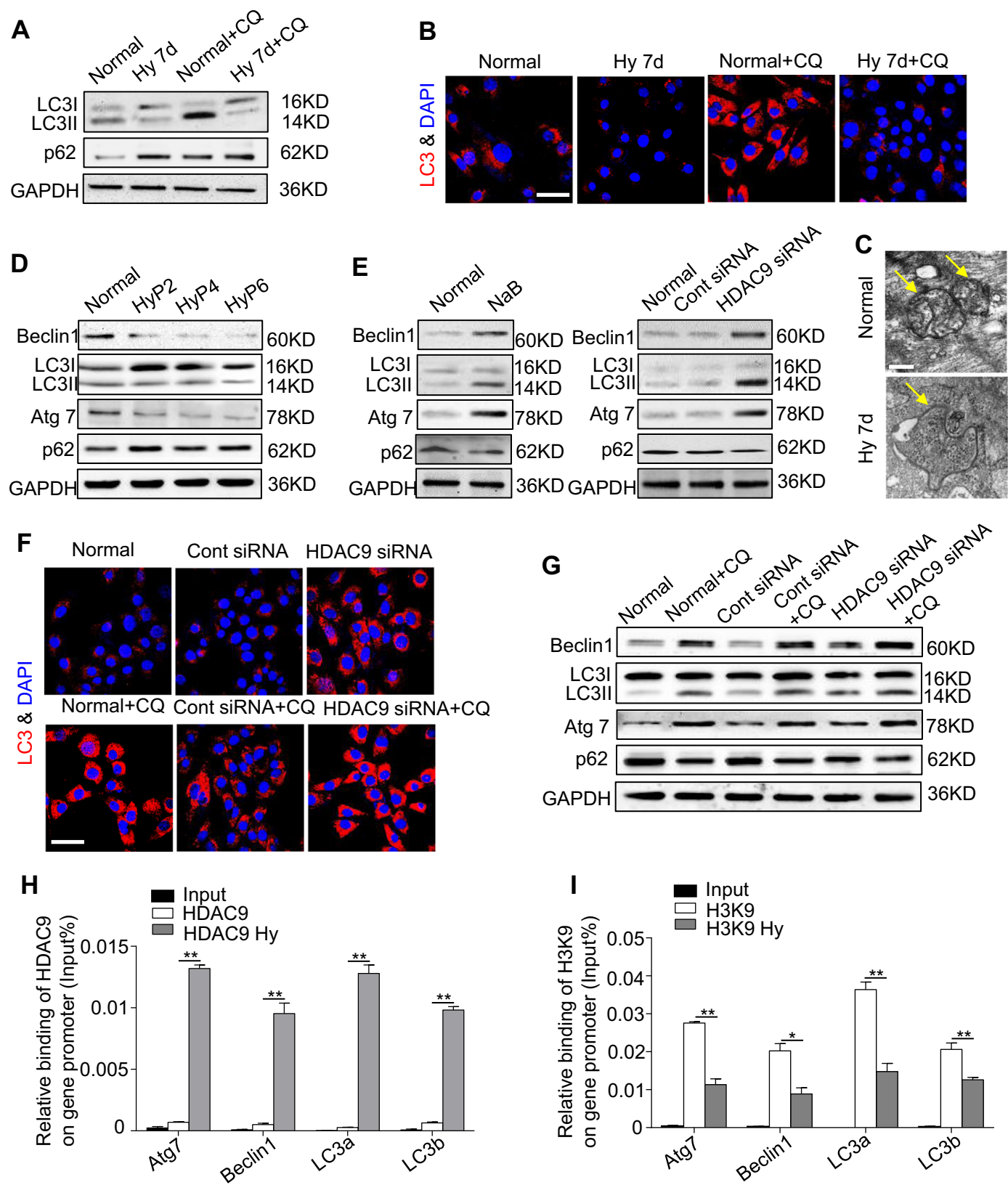

Fig. 4 HDAC9 epigenetically regulates the autophagy level in C2C12 cells. a The expression levels of the autophagy-related proteins Beclin1, LC3//II and specific cargo p62 after exposure to hypoxia for 7 days with or without chloroquine (CQ) were examined by western blotting. $\mathbf{b}$ The C2C12 cells were cultured under a normoxic or hypoxic microenvironment for 7 days with or without CQ. The LC3 (red)/nuclei (blue) in the C2C12 cells were analyzed by immunofluorescence staining. Scale bar: $50 \mu \mathrm{m}$. c To observe the autophagosomes, the $\mathrm{C} 2 \mathrm{C} 12$ cells were cultured under normoxic or hypoxic conditions for 7 days and then observed using an electron microscope. Scale bar: $2 \mu \mathrm{m}$. $\mathbf{d}$ The expression levels of autophagyrelated proteins in Hyp 2, Hyp 4, and Hyp 6 of the C2C12 cells were examined by western blotting. e The expression levels of autophagy-related proteins were examined by western blotting after treatment with NaB and HDAC9 siRNA. f, g Downregulation of HDAC9 in the normoxic C2C12 cells with or without CQ is shown. The LC3 (red)/nuclei (blue) in the C2C12 cells was analyzed by immunofluorescence staining, and the autophagyrelated genes were analyzed by western blotting. Scale bar: $50 \mu \mathrm{m} . \mathbf{h}, \mathbf{i}$ Chromatin was isolated from the C2C12 cells exposed or not to hypoxia and subjected to the chromatin immunoprecipitation assay using acetylated-histone H3K9 (Ac-H3K9) and HDAC9 antibodies. The lgG antibody was included as a control. The data are presented as the mean \pm s.d. of triplicate samples from a representative experiment. ${ }^{*} P<0.05$, ${ }^{*} P<0.01$. One-way analysis of variance (ANOVA) 
(Fig. $4 \mathrm{e}-\mathrm{g}$ ), as shown by western blotting and immunostaining analyses. These results indicate that HDAC9 and intracellular autophagy likely have a close relationship.

To examine whether HDAC9 directly regulates autophagy-related gene expression, we performed a chromatin immunoprecipitation (ChIP) assay. The results showed that HDAC9 was highly enriched at the promoters of Atg7, Beclin1, LC3a, and LC3b in the C2C12 (Fig. 4h), indicating that HDAC9 directly binds to the promoters of those autophagy-related genes. Accordingly, H3K9 was also highly enriched at the promoters of autophagy-related genes in the $\mathrm{C} 2 \mathrm{C} 12$ (Fig. 4i), indicating that HDAC9 epigenetically regulates intracellular autophagy in $\mathrm{C} 2 \mathrm{C} 12$.

Next, we tested whether the therapeutic effects of $\mathrm{NaB}$ or HDAC9 siRNA could rescue the hypoxia-impaired $\mathrm{C} 2 \mathrm{C} 12$ directly by regulating autophagy. After Beclin1 was downregulated, the autophagy level decreased significantly and then suppressed the myogenic differentiation of $\mathrm{C} 2 \mathrm{C} 12$, whereas overexpression of Beclin1 enhanced autophagy and myogenesis, as shown by qRTPCR and western blotting (Supplementary Fig. 7, Fig. 5a).
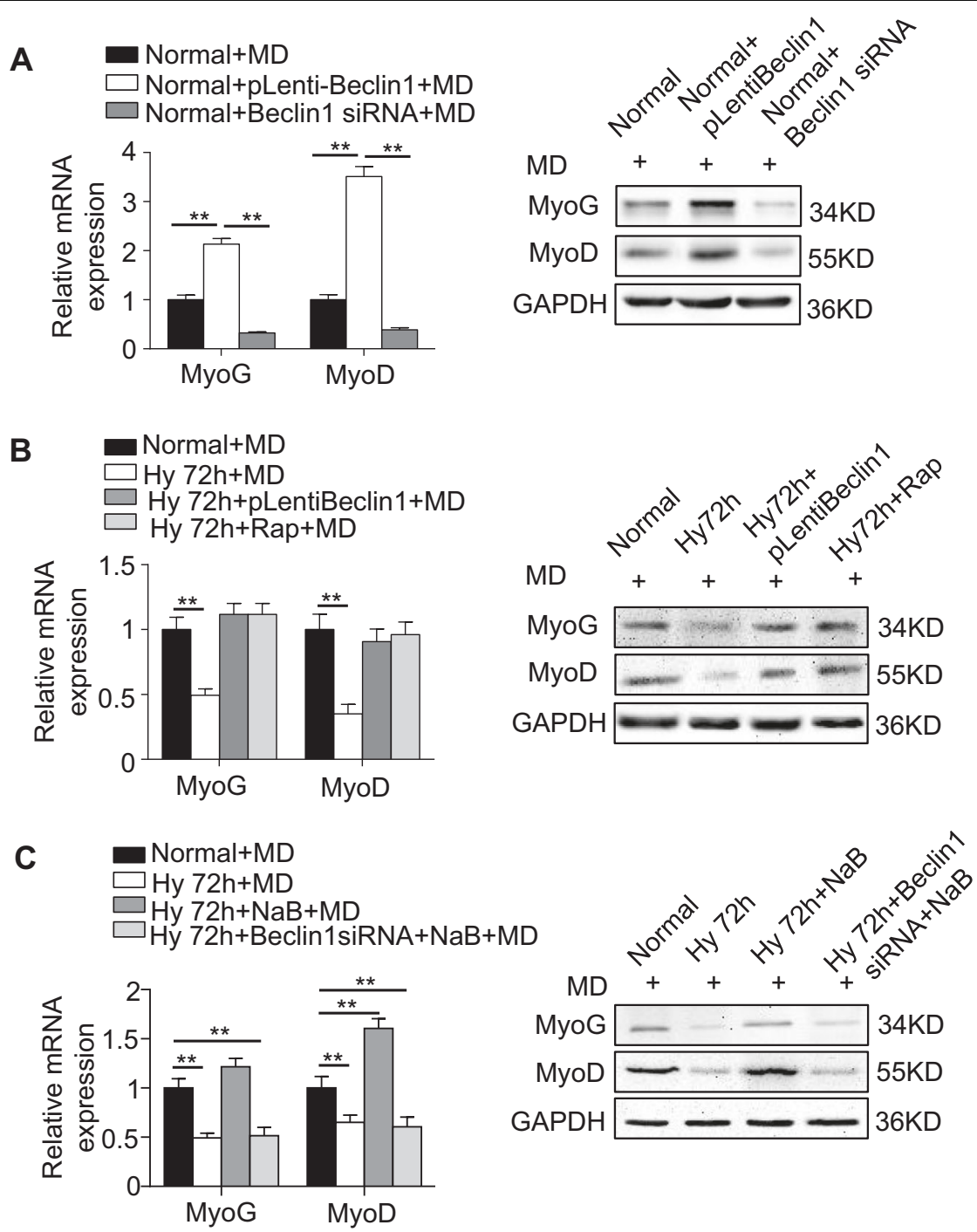

Fig. 5 HDAC9 regulates myogenic differentiation of $\mathbf{C 2 C 1 2}$ cells likely through autophagy. a The regulation of Beclin1 expression in the $\mathrm{C} 2 \mathrm{C} 12$ cells through the lentiviral vector and siRNA is depicted. The myogenesis-related genes MyoG and MyoD were examined by qRT-PCR and western blotting. b After activating autophagy in the hypoxic $\mathrm{C} 2 \mathrm{C} 12$ cells by overexpression of Beclin 1 or Rapamycin (Rap), the myogenesis-related genes MyoG and MyoD were examined by qRT-PCR and western blotting. $\mathbf{c}$ The $\mathrm{C} 2 \mathrm{C} 12$ cells were cultured in MD and treated with NaB or Beclin1 siRNA under hypoxia for $72 \mathrm{~h}$. The myogenesis-related genes MyoG and MyoD were examined in the C2C12 cells by qRT-PCR and western blotting. Normoxic $\mathrm{C} 2 \mathrm{C} 12$ cells were used as a control. The data are presented as the mean \pm s.d. of triplicate samples from a representative experiment. ${ }^{*} P<0.05,{ }^{* *} P<0.01$. One-way analysis of variance (ANOVA) 
Then, we observed that activating autophagy in the $\mathrm{C} 2 \mathrm{C} 12$ by upregulating Beclin1 or rapamycin could rescue the impaired myogenesis caused by hypoxia (Fig. 5b, Supplementary Fig. 8). More importantly, $\mathrm{NaB}$ could rescue myogenesis in the $\mathrm{C} 2 \mathrm{C} 12$ after exposure to hypoxia, but this effect could be blocked by the downregulation of Beclin1 (Fig. 5c). Together, these results reveal that hypoxia reduced the myogenesis in the $\mathrm{C} 2 \mathrm{C} 12$ mainly through HDAC9-mediated epigenetic inhibition of autophagy. We next assessed the mechanism by which autophagy regulates myogenesis.

\section{Autophagy regulates myogenesis in $\mathrm{C} 2 \mathrm{C} 12$ cells by activating the $\mathrm{Wnt} / \beta$-catenin pathway}

Wnts represent a class of secreted signaling proteins that modulate cell fate decisions, cell proliferation, and stem cell activity in a variety of embryonic and adult tissues. Many robust studies have reported that canonical Wnt signaling is important for regulating the muscle regeneration and myogenesis of myoblasts ${ }^{27,28}$. We also observed that the expression levels of phosphorylated GSK3 $\beta$ (p-GSK3 $\beta$ ) and activated $\beta$-catenin (ac- $\beta$-catenin) were both decreased after exposure to hypoxia, as shown by immunostaining and western blotting. Furthermore, the levels of the CCND1 and Axin2 mRNAs, downstream intermediates in the $\mathrm{Wnt} / \beta$-catenin pathway, were much lower in the hypoxic $\mathrm{C} 2 \mathrm{C} 12$ (Fig. $6 \mathrm{a}-\mathrm{C}$ ), suggesting that the $\mathrm{Wnt} / \beta$-catenin pathway was inactivated in hypoxia. More convincingly, we observed that activation of the canonical Wnt pathway by Wnt3a effectively rescued the impaired myogenesis in the $\mathrm{C} 2 \mathrm{C} 12$ caused by hypoxia (Supplementary Fig. 9). These data indicate that inactivation of the $\mathrm{Wnt} / \beta$-catenin pathway may contribute to the impaired function of the $\mathrm{C} 2 \mathrm{C} 12$ caused by hypoxia.

Given that emerging studies have indicated that autophagy can contribute to regulation of the canonical Wnt pathway ${ }^{29}$, we first examined the expression of $\mathrm{p}$ GSK3 $\beta$ and ac- $\beta$-catenin after regulating autophagy by lentivirus. Downregulation of Beclin1 in the normoxic C2C12 impaired the Wnt pathway, mimicking the phenotype of hypoxic $\mathrm{C} 2 \mathrm{C} 12$. In contrast, recovering Beclin1 expression in the hypoxic cells reactivated the Wnt pathway, as confirmed by western blotting and TOPflash assays (Fig. 6d, e). These results suggest that the Wnt pathway was activated or inactivated depending on the level of autophagy. More importantly, we cultured C2C12 in normoxia and hypoxia for $72 \mathrm{~h}$ and visualized p-GSK3 $\beta$ and LC3 via confocal laser scanning microscopy. The results showed that LC3 could colocalize with p-GSK3 $\beta$ under both normoxic and hypoxic conditions. Notably, the merged images demonstrated that the colocalization of p-GSK3 $\beta$ and LC3 was lower in the hypoxic cell group due to the decreased expression levels of p-GSK3 $\beta$ and
LC3 (Fig. 6f). Collectively, these results indicate that autophagy could directly regulate the canonical Wnt pathway, likely via phosphorylated GSK3 $\beta$.

We then investigated whether autophagy regulates myogenic differentiation through the $\mathrm{Wnt} / \beta$-catenin pathway. The results indicated that knockdown of the expression of $\beta$-catenin reduced the myogenesis in C2C12. Importantly, rapamycin could not recover the inhibited myogenesis after $\beta$-catenin was downregulated (Supplementary Fig. 10, Fig. 6g). Finally, to confirm the regulatory network of histone deacetylase, autophagy, and the canonical Wnt pathway, the $\mathrm{C} 2 \mathrm{C} 12$ were treated with $\mathrm{NaB}$, 3-MA, and DKK-1. Notably, $\mathrm{NaB}$ inhibited the expression of HDAC9 and promoted the expression of the remaining downstream genes, whereas the autophagy inhibitor 3-MA inhibited the expression of Beclin1 and ac- $\beta$-catenin. Furthermore, the canonical Wnt pathway inhibitor DKK-1 blocked only the expression of ac$\beta$-catenin (Fig. 6h). Together, these results imply that the canonical Wnt pathway is under downstream regulation by HDAC9-mediated autophagy.

\section{HDAC9 epigenetically regulated autophagy is also observed in ischemic muscle atrophy}

To further verify our investigation conducted in vitro, we first developed an ischemic muscle atrophy model in mice. The sections from mouse gastrocnemius muscles were examined histologically to evaluate the effects of chronic ischemic hypoxia on tissue integrity. The results showed normoxically grown muscle fibers had uniform size and shape with peripherally placed nuclei (Fig. 7a). Muscle tissue from mice exposed to ischemic hypoxia for 21 days showed severe atrophy of muscle fibers with irregularity in fiber size and more space between them. However, no necrotic fiber or cell splitting could be observed in any of the micrographs (Fig. 7a). Furthermore, with increased duration of exposure to ischemic hypoxia, the ratio of rat gastrocnemius muscle weight/tibial length decreased significantly, decreasing by $22 \%$ during 21 days of hypoxia exposure (Fig. 7b). Calpains are a member of the $\mathrm{Ca}^{2+}$-activated cysteine proteases that play important roles in cell motility, cell proliferation, and apoptosis ${ }^{30}$. Some investigations have suggested that calpains are activated during atrophy ${ }^{31,32}$. In our study, we observed that the expression level of calpain increased obviously after exposure to hypoxia for 21 days (Fig. 7c), suggesting that the muscle is atrophied after exposure to chronic ischemic hypoxia.

Given that HDAC9 expression was increased in the $\mathrm{C} 2 \mathrm{C} 12$ after exposure to hypoxia and subsequently affected cell functions via the regulation of autophagy, we next tested whether HDAC9 regulates the level of autophagy in an ischemic hypoxia mouse model. The 

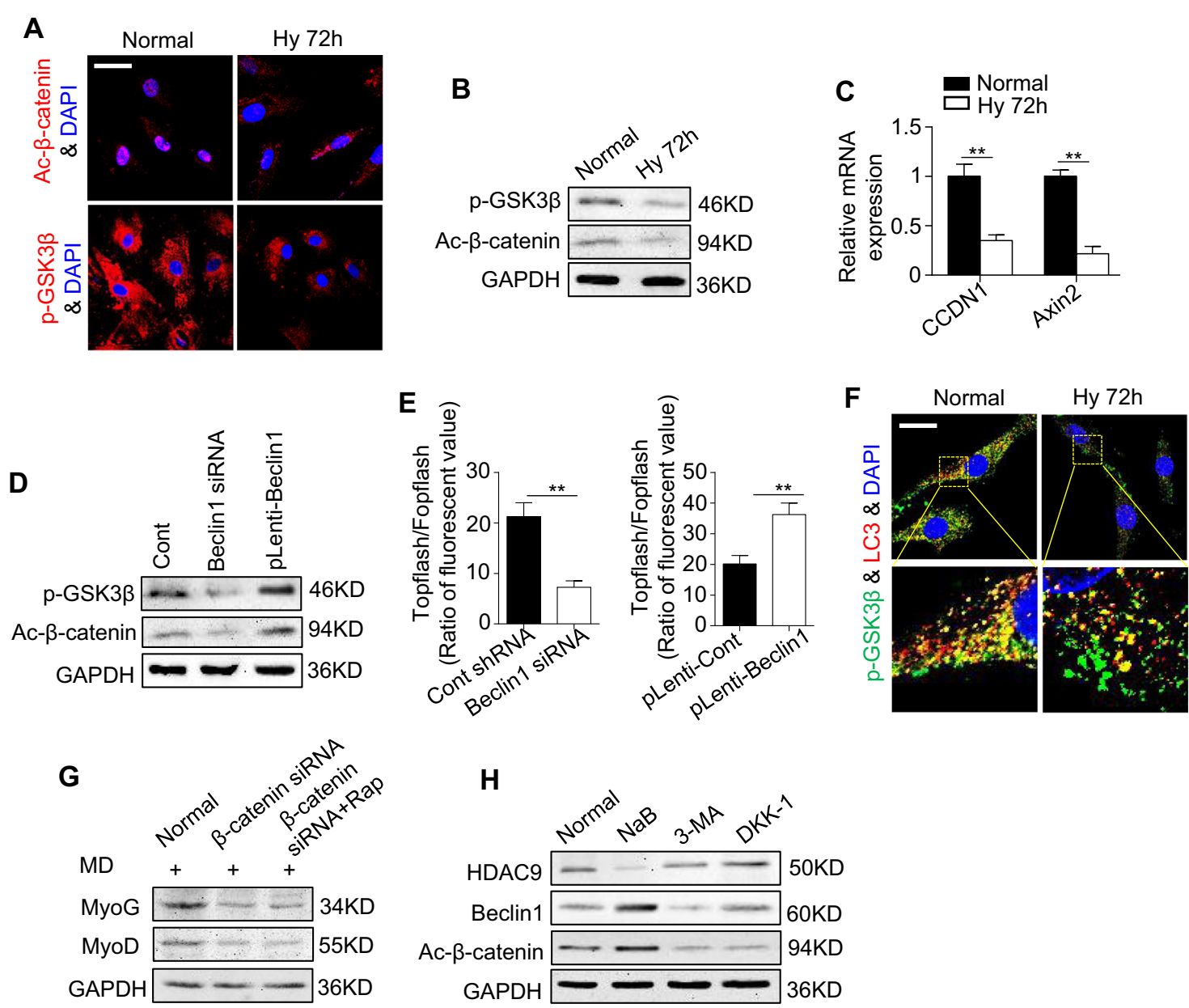

Fig. 6 Autophagy regulates myogenic differentiation in $\mathrm{C} 2 \mathrm{C} 12$ cells through regulation of the canonical Wnt pathway. a-c The expression levels of p-GSK3 $\beta$ and active- $\beta$-catenin were examined by immunofluorescence staining (a) and western blotting (b) after the cells were cultured in normoxia or hypoxia for $72 \mathrm{~h}$. The downstream genes of the canonical Wnt pathway were analyzed by qRT-PCR (c). Scale bar: $50 \mu \mathrm{m}$. d The expression levels of $\mathrm{p}$-GSK3 $\beta$ and active- $\beta$-catenin in the C2C12 cells were examined by western blotting after downregulation of Beclin1. e Activation of the canonical Wnt pathway was examined $48 \mathrm{~h}$ after transfection by luciferase assay. $\mathbf{f}$ Immunostaining showed overlapping of LC3 (red) and p-GSK3 3 (green) in the C2C12 cells cultured in normoxia and hypoxia for $72 \mathrm{~h}$. Scale bar: $25 \mu \mathrm{m}$. g C2C12 cells were cultured in MD and transfected with control siRNA or $\beta$-catenin siRNA, and rapamycin was used to activate autophagy. Myogenesis-related genes were examined by western blotting after treatment for $72 \mathrm{~h}$. $\mathbf{h}$ The $\mathrm{C} 2 \mathrm{C} 12$ cells were treated with NaB, 3-MA, and DKK-1. The expression levels of HDAC9, Beclin1, and ac$\beta$-catenin were examined by western blotting. The data are presented as the mean \pm s.d. of triplicate samples from a representative experiment. ${ }^{*} P<0.05,{ }^{* *} P<0.01$. c One-way analysis of variance (ANOVA). e unpaired two-tailed Student's $t$-test

expression of HDAC9 increased but the level of H3K9ac decreased in the ischemic hypoxia group, as shown by western blotting (Fig. 7d). As expected, autophagy was dramatically lost in the hypoxia mouse model, as indicated by decreased LC3 levels (Fig. 7e), as in the C2C12 cultured in hypoxia. More importantly, we also observed the same muscle atrophy and decreased autophagy levels in patients with chronic muscle atrophy caused by arteriosclerosis obliterans (Fig. 7f, g). Collectively, our results indicate that hypoxia inhibits the regeneration of muscle likely via autophagy that is epigenetically regulated by HDAC9 (Fig. 8).

\section{Discussion}

The effect of hypoxia on the myogenic process has been extensively studied. Several studies have provided credible data to show that less than $1 \% \mathrm{O}_{2}$ level represses the proliferation and myogenesis of primary myoblasts and $\mathrm{C} 2 \mathrm{C} 12$ in vitro $^{2,6,33}$, raising the question of whether a long-term hypoxic microenvironment could permanently impair the myogenesis process. Some studies have demonstrated that myoblast differentiation is reversibly inhibited by hypoxia ${ }^{2}$, as myoblasts regain myogenesis capability when normal oxygen levels are restored. Conversely, our results revealed that the inhibitory effect on 

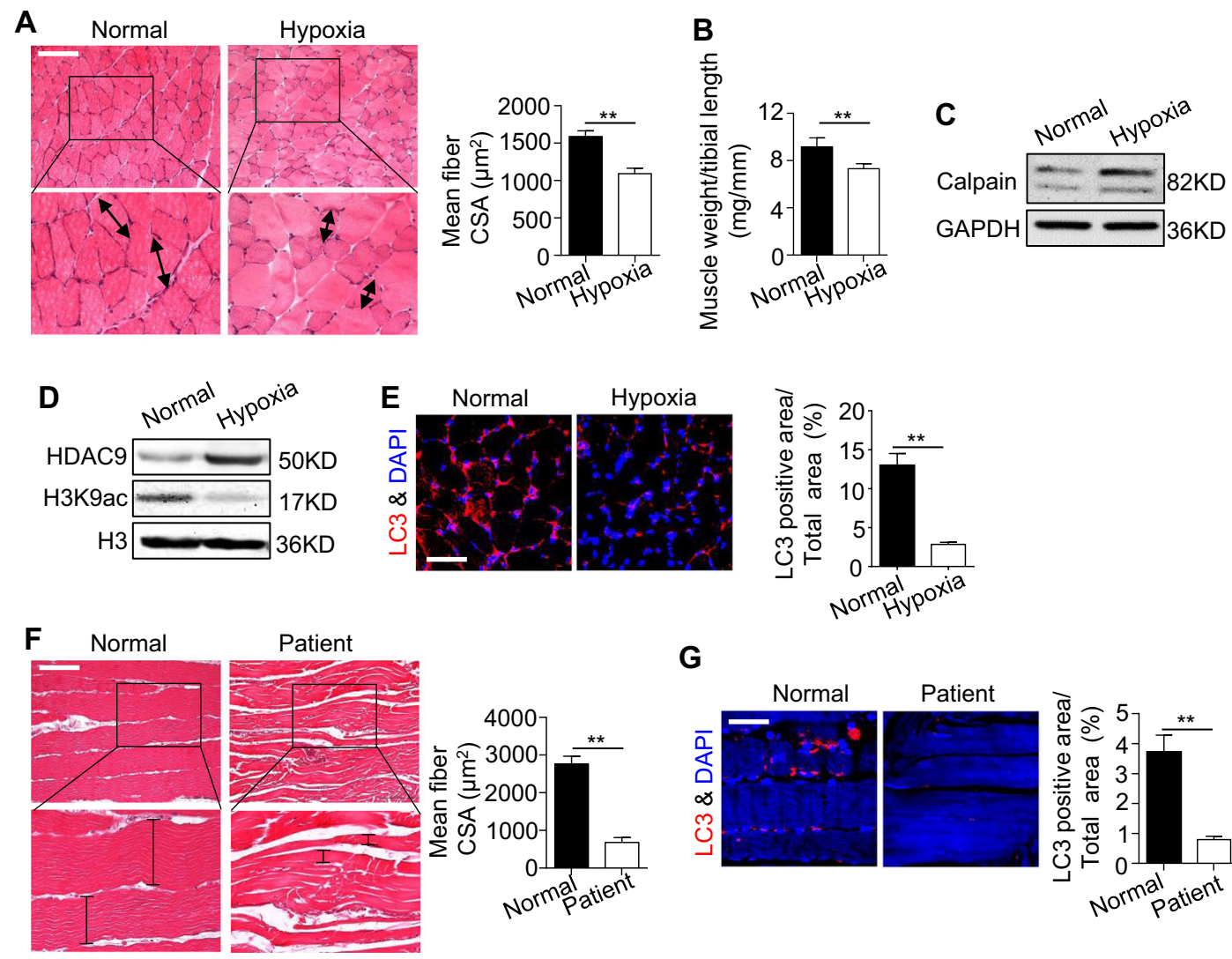

G

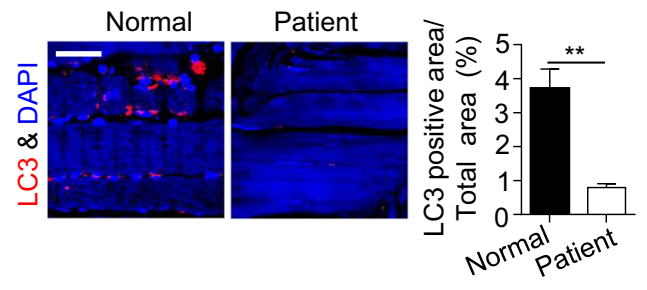

Fig. 7 The autophagy that is epigenetically regulated by HDAC9 is also observed in the femoral artery (FA) ligation model and arteriosclerosis obliterans patients. We constructed a mouse single FA ligation model and harvested samples from both the control and surgery groups on day 21. a Hematoxylin and eosin staining (h\&e) was used for visualizing the tissue from the mouse gastrocnemius muscle of the FA ligation model. The results from the quantitative analysis of the muscle fiber is indicated in the right panel. Scale bar: $200 \mu \mathrm{m}$. b, c The ratio of muscle weight/tibial length (b) and calpain expression (c) were analyzed in the control and ligation groups. $\mathbf{d}$ The expression levels of HDAC9 and H3K9 in the two groups were examined by western blotting. e The muscles samples were immunostained using anti-LC3 (red) and nuclear staining (blue, DAPI) to reveal the autophagy level. The results from the quantification analysis of the LC3 ${ }^{+}$cells is shown in the right panel. Scale bar: $100 \mu \mathrm{m}$. $\mathbf{f} \mathrm{H \& E}$ staining of the muscle in the normal control and arteriosclerosis obliterans patients is shown. The results from the quantification analysis are shown in the right panel. Scale bar: $200 \mu \mathrm{m}$. $\mathbf{g}$ The immunostaining analysis showed the expression of LC3 (red) and DAPI (blue) in the distal gastrocnemius muscle. Quantification of the LC3 ${ }^{+}$cells is indicated in the right panel. Scale bar: $100 \mu \mathrm{m} . n=8$ for the mouse model and $n=2$ for the patients. The data are presented as the mean \pm s.d. of triplicate samples from a representative experiment. ${ }^{*} P<0.05$, ${ }^{* *} P<0.01$. Unpaired two-tailed Student's $t$-test

myogenesis caused by hypoxia was sustained in the $\mathrm{C} 2 \mathrm{C} 12$ after six passages when normal oxygen was restored. We think the difference between the results from previous studies and those from our study may be due to the different culture conditions and observation methods. We previously reported that the microenvironment induces long-term effects on cell functions due to epigenetic regulation ${ }^{5}$. A previous report demonstrated that hypoxia-induced myogenic differentiation of embryonic stem cells is mediated by regulating HDAC $6^{34}$. Another study has also shown that myogenesis under hypoxia is correlated with the deacetylation of histones associated with the myoD promoter ${ }^{33,35-37}$. Therefore, we first screened 11 HDACs. The results showed that HDAC9 was significantly upregulated in response to hypoxia. Downregulation of HDAC9 by applying
HDAC9 siRNA or the HDACs inhibitor $\mathrm{NaB}$ recovered the inhibitory effect of hypoxia on $\mathrm{C} 2 \mathrm{C} 12$ myogenesis. Thus, we showed that chronic exposure to severe hypoxia-induced muscle dysfunction or mass loss likely through epigenetic regulation similar to that observed in hypoxia-induced Alzheimer's disease ${ }^{38,39}$, pulmonary hypertension ${ }^{40}$, and cardiac tissue fibrosis ${ }^{41}$. Notably, we observed that the autophagy of the $\mathrm{C} 2 \mathrm{C} 12$ was first enhanced after exposure to hypoxia and subsequently decreased after $6 \mathrm{~h}$ in hypoxic culture. However, the expression of HDAC9 was continuously increased after exposure to hypoxia. Therefore, we infer that HDAC9 likely regulates autophagy during long-term hypoxic exposure.

Autophagy ${ }^{24,42}$ is a primary survival pathway for recycling cellular material during periods of nutrient 


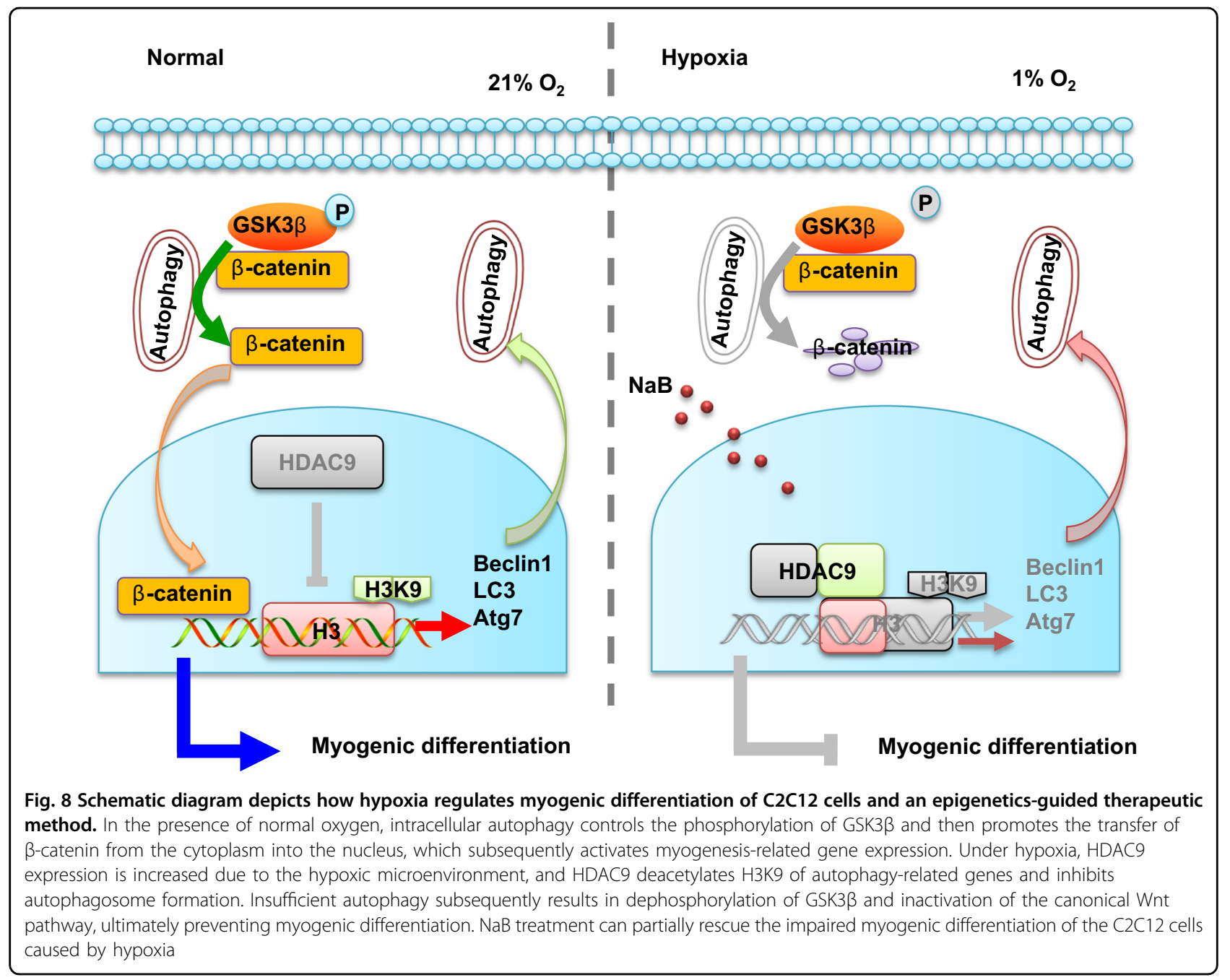

starvation and in response to hypoxia, endoplasmic reticulum stress, and other stresses, and it is essential for maintaining tissue homeostasis. Importantly, dysregulated autophagy is observed in the pathogenesis of myopathies and muscular dystrophies ${ }^{43,44}$. A recent study demonstrated that autophagy was significantly enhanced in response to short-term intermittent hypoxia-induced atrophy of skeletal muscle ${ }^{45}$. In that study, LC3II protein was increased (2.4-fold) in limb muscle after 4 days of intermittent exposure to hypoxia. However, the effect of hypoxia-induced autophagy on myoblast differentiation has not been well elucidated. In the present work, we found that long-term exposure to hypoxia reduced the level of autophagy in the $\mathrm{C} 2 \mathrm{C} 12$ and that autophagy was suppressed with cell passages. However, we noticed that the level of autophagy in the $\mathrm{C} 2 \mathrm{C} 12$ was increased significantly under a short-term $(<6 \mathrm{~h})$ hypoxic stimulus and then gradually declined with prolonged hypoxia (data not shown). This finding is in agreement with previous reports showing that acute exposure to hypoxia increases the expression of markers of autophagy ${ }^{45,46}$. Interestingly, we noted that the inhibition of autophagy by Beclin1 siRNA blocked the effect of the $\mathrm{NaB}$ on rescuing the function of the $\mathrm{C} 2 \mathrm{C} 12$ in hypoxia. Importantly, we confirmed that HDAC9 directly bound to the promoters of Atg7, Beclin1, LC3a, and LC3b in C2C12. The abovementioned results suggest that autophagy, which is epigenetically regulated by HDAC9, is required to maintain the function of $\mathrm{C} 2 \mathrm{C} 12$ in a chronic hypoxic microenvironment.

Some studies have shown that $\mathrm{Wnt} / \beta$-catenin signaling is critical for the regulation of muscle development and the myogenic differentiation of myoblasts ${ }^{47-49}$. Conditional depletion of $\beta$-catenin in mice led to reduced muscle mass and fewer myofibers, as shown by Pax7positive muscle progenitor cell staining ${ }^{50}$. Previous reports have demonstrated that $\mathrm{Wnt} / \beta$-catenin signaling is essential for the multiple steps of myogenesis ${ }^{28,48,51,52}$, but few reports have indicated that $\mathrm{Wnt} / \beta$-catenin signaling is not required during muscle regeneration ${ }^{53}$. In 
the present study, we revealed that autophagy maintained the functions of myoblast cell lines by regulating the phosphorylation of GSK3 $\beta$, which is an important regulator for modulating $\beta$-catenin nuclear translocation in hypoxia. Although many researchers have demonstrated that GSK3 $\beta$ determines the activation of autophagy during different cytological processes ${ }^{54-56}$, autophagy-regulating GSK3 $\beta$ phosphorylation has been poorly described. Recently, some studies have shown that GSK3 $\beta$ phosphorylation is suppressed by Beclin1 siRNA in renal cells ${ }^{57}$. In combination with our results, we suggest that autophagy directly regulates the phosphorylation of GSK3 $\beta$ and thereby the activation of the Wnt/ $\beta$-catenin pathway, which is critical for maintaining the myogenesis of $\mathrm{C} 2 \mathrm{C} 12$.

Our data demonstrate that epigenetic regulation is critical for the sustained hypoxia-induced inhibitory effect on the myogenesis of myoblasts and that the activation of autophagy is a key step in rescuing the myogenic differentiation of myoblasts, which may provide a prospective strategy for treating myopathies caused by chronic hypoxia. Further studies are needed to explore how autophagy degrades phosphorylated GSK3 $\beta$.

\section{Materials and methods}

\section{C2C12 cell line culture and cell hypoxic model}

Immortalized murine $\mathrm{C} 2 \mathrm{C} 12$ myoblasts obtained from the Cell Bank of the Chinese Academy of Sciences (Shanghai, China), originally derived from $\mathrm{C} 3 \mathrm{H}$ mouse leg muscle, were cultured on uncoated 6-well plates at $37^{\circ} \mathrm{C}$ and $5 \% \mathrm{CO}_{2}$ in high-glucose growth medium $(4.5 \mathrm{~g} / \mathrm{l} \mathrm{d}-$ glucose) DMEM (GIBCO-BRL, Gaithersburg, MD, USA) supplemented with $10 \%$ fetal bovine serum (FBS, GIBCOBRL), $2 \mathrm{mM}$ L-glutamine (Life Technologies, Rockville, $\mathrm{MD}$, USA), and $1 \%$ penicillin-streptomycin (GIBCO$\mathrm{BRL}$ ). After reaching confluence, $\mathrm{C} 2 \mathrm{C} 12$ myoblasts were induced in differentiation medium (DMEM supplemented with $2 \%$ horse serum, $1 \mathrm{mM}$ L-glutamine, $1 \%$ penicillin-streptomycin) for 2-3 days.

To establish hypoxia culture conditions, $\mathrm{C} 2 \mathrm{C} 12$ myoblasts were placed in a hypoxic $\left(1 \% \mathrm{O}_{2}, 5 \% \mathrm{CO}_{2}, 37^{\circ} \mathrm{C}\right)$ incubator (Galaxy oxygen control incubator, RS Biotech, Irvine, UK) for 1, 3, 6, 12, 24, $72 \mathrm{~h}$ and 7 days. Control cells (Normal group) were incubated for equivalent time frames under normoxic conditions $\left(21 \% \mathrm{O}_{2}, 5 \% \mathrm{CO}_{2}, 37^{\circ} \mathrm{C}\right)$.

\section{MTT assay}

The viability of $\mathrm{C} 2 \mathrm{C} 12$ myoblasts cultured under normoxic or hypoxic conditions was determined using a 3-(4,5-dimethylthiazol-2yl)-2,5-diphenyltetrazolium bromide (MTT) assay carried out for 8 days according to the manufacturer's protocol (Sigma-Aldrich). Absorbance was determined at $490 \mathrm{~nm}$ with a microplate reader (BioTek Instruments, Winooski, VT, USA). Experiments were performed in triplicate.

\section{5-Ethynyl-2'-deoxyuridine assays}

The proliferation of $\mathrm{C} 2 \mathrm{C} 12$ myoblasts cultured under normoxic or hypoxic conditions was determined using an 5-Ethynyl-2'-deoxyuridine (EdU) DNA Proliferation in vitro Detection kit (RiboBio, Guangzhou, China) according to manufacturer's instructions. Flow cytometry was performed on these cells previously labeled with EdU.

\section{Apoptosis analysis}

The apoptosis of $\mathrm{C} 2 \mathrm{C} 12$ myoblasts cultured under normoxic or hypoxic conditions was determined using an apoptosis detection kit (BD Pharmingen) according to the manufacturer's instructions. Flow cytometry was performed to analyze cell apoptosis, discriminating viable, dead, early apoptotic and late apoptotic cells, by detecting the Annexin-V and propidium iodide (PI) staining and comparing the percentages with the numbers determined for the control group.

\section{Immunofluorescence staining}

$\mathrm{C} 2 \mathrm{C} 12$ cells cultured under normoxic or hypoxic conditions until confluence were subsequently maintained in differentiation medium for 3 days to promote myoblast fusion. The cells were then fixed with $4 \%$ paraformaldehyde for $15 \mathrm{~min}$ at $4{ }^{\circ} \mathrm{C}$, permeabilized with $0.2 \%$ Triton X-100 in PBS for $10 \mathrm{~min}$ and blocked in 5\% normal goat serum for $30 \mathrm{~min}$. For immunofluorescence staining, C2C12 cells were incubated with Myosin (R\&D system, 1:100, Minneapolis, MN, USA, MAB4470) or LC3I/II (Cell signal, 1:100, 12741) primary antibodies overnight at $4{ }^{\circ} \mathrm{C}$. Subsequently, they were incubated with Cy3-/FITCsecondary antibody for $1 \mathrm{~h}$ at room temperature according to the manufacturer's instructions.

\section{qRT-PCR analysis}

Total mRNA isolated from $\mathrm{C} 2 \mathrm{C} 12$ cells using TRIzol (Invitrogen, Carlsbad, CA, USA) according to the manufacturer's instructions was reverse-transcribed into cDNA. Real-time PCR detection was carried out using Primescript $^{\mathrm{TM}} \mathrm{RT}$ master mix (Takara Bio Inc., Otsu, Japan), SYBR Premix Ex TaqTMII (Takara Bio Inc.), and the CFX96 Trademark Real-time PCR detection system (BioRad, California, USA). The expression levels of $M y o G$, MyoD, HDAC1-11, LC3, Beclin1, Atg7, Axin2, and $C C N D 1$ were examined with the primers (Sangon Biotech, China) listed in Supplementary Table 1. GAPDH served as a housekeeping gene. Experiments were performed in triplicate.

\section{Western blotting analysis}

C2C12 cells were harvested in RIPA lysis buffer (Beyotime Institute of Biotechnology, Shanghai, China). Whole-cell protein extracts were quantified using the BCA assay, separated by SDS-PAGE $8-12 \%$, and then 
transferred to PVDF membranes (Millipore, Billerica, MA, USA). Antibodies included Myogenin (Abcam, 1:1000, ab124800, Cambridge, MA, USA), MyoD (Santa Cruz, 1:200, sc-377460), HDAC9 (Abcam, 1:1000, ab59718), HIF1 $\alpha$ (Abcam, 1:1000, ab179483), HIF2 $\alpha$ (Abcam, 1:1000, ab179825), H3K9 (Abcam, 1:1000, ab32129), H3K14, H3K18, H4K16 (Cell Signaling, 1:1000), LC3I/II (Cell Signal, 1:1000, 12741), Beclin1 (Cell Signal, 1:1000, 3738), Atg5 (Cell Signal, 1:1000, 12994), Atg7 (Cell Signal, 1:1000, 8558), Atg12 (Cell Signal, 1:1000, 4180), p62 (Cell Signaling, 1:1000, 23214), p-GSK3 $\beta$ Ser9 (Cell Signal, 1:1000, 9323), GSK3 $\beta$ (Cell Signal, 1:1000, 12456), and active- $\beta$-catenin (Millipore, 1:800, 05-665). Stripped membranes were reprobed with GAPDH (Abcam, 1:4000, ab181602) as a loading control. Signal detection was performed using the ECL Kit (Beyotime Institute of Biotechnology) after incubation with an anti-rabbit or antimouse IgG secondary antibody (CoWin Bioscience Co., Beijing, China). Experiments were performed in triplicate.

\section{Transfection assay}

siRNA duplex oligonucleotides against mouse HDAC9 (Gene-Pharma Co, Shanghai, China), $\beta$-catenin (GenePharma Co, Shanghai, China), Beclin1 (Gene-Pharma Co), or the negative control (Gene-Pharma $\mathrm{Co}$ ) were transfected into both normoxic and hypoxic $\mathrm{C} 2 \mathrm{C} 12$ myoblasts at a final concentration of $50 \mathrm{nM}$ using siPORTNeoFX. The medium was replaced $8 \mathrm{~h}$ later. Experiments were performed in triplicate.

\section{Small molecule administration}

To examine the effect of sodium butyrate $(\mathrm{NaB})$ on the myogenesis of $\mathrm{C} 2 \mathrm{C} 12$ cells, $\mathrm{C} 2 \mathrm{C} 12$ cells were cultured under hypoxia with or without $\mathrm{NaB}(200 \mu \mathrm{M}$, sigma, 15654-7). To examine the effect of $\mathrm{NaB}$ and Trichostatin $\mathrm{A}$ (TSA) on autophagy of $\mathrm{C} 2 \mathrm{C} 12$ cells, $\mathrm{C} 2 \mathrm{C} 12$ cells were cultured under normoxic conditions with or without $\mathrm{NaB}$ $(200 \mu \mathrm{M})$ and TSA (100 nM, Sigma, 58880-19-6). Rapamycin (100 nM, Sigma, 53123-88-9), NaB (200 $\mu$ M), 3MA (5 mM, Sigma, 5142-23-4), and recombinant mouse DKK-1 (100 ng/ml, BioLegend, San Diego, CA, USA, 759604) were added to $\mathrm{C} 2 \mathrm{C} 12$ cells cultured under normoxic or hypoxic conditions to examine the relationship between autophagy and the signaling pathways. All the cell samples for qRT-PCR and western blotting were collected according to the manufactures' instructions.

\section{Transmission electron microscope (TEM) analysis}

C2C12 cells were collected with trypsin, washed with serum-free PBS and primarily fixed in $4 \%$ glutaraldehyde and $4 \%$ paraformaldehyde (Sigma, $\mathrm{pH}$ 7.2) overnight. After washing with PBS, the cells were progressively dehydrated in a graduated series of ethanol solutions (50, 70,95 , and $100 \%$ ), and then embedded in situ in LX-812 resin (Ladd Research Industries Inc., USA). Subsequently, ultrathin sections $(60 \mathrm{~nm})$ were stained with $1 \%$ uranyl acetate $(30 \mathrm{~min})$ and lead citrate $(10 \mathrm{~min})$. The ultrastructure of the cells was then observed using an FEI Tecnai G12 Spirit BioTwin transmission electron microscope (FEI Company, USA) with an accelerating voltage of $100 \mathrm{kV}$. Digital images were captured on a Veleta CCD camera (Olympus-SIS, Germany).

\section{Chromatin immunoprecipitation}

We used a Chromatin immunoprecipitation (ChIP) assay kit (Merck Millipore, Billerica, MA, USA, 17-371) to confirm the binding between proteins and gene promoters according to the manufacturer's protocol. Antibodies against HDAC9 (Abcam, ab59718) and polyclonal anti-Histone H3 (acetyl K9) (Abcam, ab10812) were used as detection antibodies, and normal rabbit IgG (Merck Millipore) was used as a negative control. All precipitated DNA samples were analyzed by qRT-PCR, and the results were normalized to the input value. The primers spanning the H3K9-/HDAC9-binding sites at the Atg7, Beclin1, $L C 3 a$, and $L C 3 b$ promoter (Sangon biotech, China) are listed in Supplementary Table 2.

\section{Hind limb ischemia model}

Male 4-month mice were purchased from the Animal Center of Fourth Military Medical University, Xi'an, China. Sixteen mice were randomly and evenly divided into two groups (eight in each group) to receive either sham or femoral artery ligation surgery. Briefly, femoral artery was ligated at the proximal region just under the inguinal ligament and distally above the profunda femoris branch. A cut was then made between the ligation sites. All of the procedures that involved animals were approved by the Animal use and care committee of the Fourth Military Medical University (license number: SYXK 20120023).

\section{Human subjects}

Two arteriosclerosis obliteran patients (male), aged 48 and 53 years respectively were conducted by the Affiliated Hospital of Fourth Military Medical University because of their arteriosclerosis obliterans. Health human muscle samples were collected from two bone fracture patients caused by car accident aged 46 and 50 (male).

The clinical study was approved by the Ethics Committee of the Affiliated Hospital of Fourth Military Medical University, and written informed consent was obtained from all participants prior to sample collection.

\section{Statistics}

The data are presented as the mean \pm s.d. Unpaired twotailed Student's $t$-tests were applied for comparisons between two groups, and one-way analysis of variance 
(ANOVA) with a Bonferroni post-test was used for multiple comparisons. All experiments were repeated more than three times, and representative experiments are shown. $P$ values $<0.05$ were considered significant. ${ }^{*} P<0.05,{ }^{* *} P<0.01$. Analytic tests were undertaken using SPSS17.0 software.

\section{Acknowledgements}

This work was financially supported through grants from the by National Natural Science Foundation of China (31571532), Key R \& D Plan projects of Shaanxi Province (2017ZDXM-SF-038) and National Natural Science Foundation of China (81271176 and 31601099).

\section{Author details}

'General Surgery Department, Tang Du Hospital, Fourth Military Medical University, 710032 Xi'an, Shaanxi, China. ${ }^{2}$ State Key Laboratory of Military Stomatology \& National Clinical Research Center for Oral Diseases \& Shaanxi International Joint Research Center for Oral Diseases, Center for Tissue Engineering, School of Stomatology, Fourth Military Medical University, 710032 Xi'an, Shaanxi, China. ${ }^{3}$ State Key Laboratory of Military Stomatology \& National Clinical Research Center for Oral Diseases \& Shaanxi International Joint Research Center for Oral Diseases, Department of preventive dentistry, Fourth Military Medical University, 710032 Xi'an, Shaanxi, China. ${ }^{4}$ Department of Aerospace Biodynamics, Fourth Military Medical University, 710032 Xi'an, Shaanxi, China. ${ }^{5}$ State Key Laboratory of Military Stomatology \& National Clinical Research Center for Oral Diseases \& Shaanxi International Joint Research Center for Oral Diseases, Department of Orthodontics, School of Stomatology, Fourth Military Medical University, 710032 Xi'an, Shaanxi, China

\section{Author contributions}

Z.Z. collected and organized the data. L.Z. and Y.Z. wrote the most manuscript and helped analyze the data. L.L. helped with the statistical design and ChIP analysis. J.Z. and Q.W. contributed to the $\mathrm{C} 2 \mathrm{C} 12$ cell culture and identification. Z.J. designed the experiments and analyzed the data. W.L. developed the concept, supervised the project, conceived the experiments and revised the manuscript.

\section{Conflict of interest}

The authors declare that they have no conflict of interest.

\section{Publisher's note}

Springer Nature remains neutral with regard to jurisdictional claims in published maps and institutional affiliations.

Supplementary Information accompanies this paper at (https://doi.org/ 10.1038/s41419-019-1763-2).

Received: 21 March 2019 Revised: 11 June 2019 Accepted: 19 June 2019 Published online: 18 July 2019

\section{References}

1. Slot, I. G., Schols, A. M., De Theije, C. C., Snepvangers, F. J. \& Gosker, H. R. Alterations in skeletal muscle oxidative phenotype in mice exposed to 3 weeks of normobaric hypoxia. J. Cell Physiol. 231, 377-392 (2016).

2. Di Carlo, A. et al. Hypoxia inhibits myogenic differentiation through accelerated MyoD degradation. J. Biol. Chem. 279, 16332-16338 (2004).

3. Jash, S. \& Adhya, S. Effects of transient hypoxia versus prolonged hypoxia on satellite cell proliferation and differentiation in vivo. Stem Cells Int. 2015, 961307 (2015).

4. Turan, N. et al. A systems biology approach identifies molecular networks defining skeletal muscle abnormalities in chronic obstructive pulmonary disease. PLoS Comput. Biol. 7, e1002129 (2011).

5. Demonbreun, A. R. \& McNally, E. M. Muscle cell communication in development and repair. Curr. Opin. Pharmacol. 34, 7-14 (2017).
6. Wang, C. et al. Hypoxia inhibits myogenic differentiation through p53 proteindependent induction of Bhlhe40 protein. J. Biol. Chem. 290, 29707-29716 (2015).

7. Hidalgo, M. et al. Oxygen modulates the glutathione peroxidase activity during the L6 myoblast early differentiation process. Cell Physiol. Biochem. 33, 67-77 (2014)

8. Majmundar, A. J. et al. O(2) Regulates skeletal muscle progenitor differentiation through phosphatidylinositol 3-kinase/AKT signaling. Mol. Cell Biol. 32, 36-49 (2012).

9. Li, B. et al. GCN5 modulates osteogenic differentiation of periodontal ligament stem cells through DKK1 acetylation in inflammatory microenvironment. Sci. Rep. 6, 26542 (2016).

10. Dawson, M. A. \& Kouzarides, T. Cancer epigenetics: from mechanism to therapy. Cell. 150, 12-27 (2012).

11. Safronova, O. S., Nakahama, K. \& Morita, I. Acute hypoxia affects P-TEFb through HDAC3 and HEXIM1-dependent mechanism to promote genespecific transcriptional repression. Nucleic. Acids Res. 42, 8954-8969 (2014).

12. Bricambert, J. et al. Impaired histone deacetylases 5 and 6 expression mimics the effects of obesity and hypoxia on adipocyte function. Mol. Metab. 5, 1200-1207 (2016)

13. Koyuncuoglu, T. et al. Uridine protects against hypoxic-ischemic brain injury by reducing histone deacetylase activity in neonatal rats. Restor. Neurol. Neurosci. 33, 777-784 (2015).

14. To, M. et al. Defect of adaptation to hypoxia in patients with COPD due to reduction of histone deacetylase 7 . Chest 141, 1233-1242 (2012).

15. Blondelle, J. et al. HACD1, a regulator of membrane composition and fluidity, promotes myoblast fusion and skeletal muscle growth. J. Mol. Cell Biol. 7, 429-440 (2015).

16. Micheli, L. et al. PC4/Tis7/IFRD1 stimulates skeletal muscle regeneration and is involved in myoblast differentiation as a regulator of MyoD and NF-kappa B. J. Biol. Chem. 286, 5691-5707 (2011).

17. Ren, H. X., Accili, D. \& Duan, C. M. Hypoxia converts the myogenic action of insulin-like growth factors into mitogenic action by differentially regulating multiple signaling pathways. Proc. Natl Acad. Sci. USA. 107, 5857-5862 (2010).

18. De Meireles, L. C. et al. Treadmill exercise induces selective changes in hippocampal histone acetylation during the aging process in rats. Neurosci. Lett. 634, 19-24 (2016).

19. Villagra, A., Sotomayor, E. M. \& Seto, E. Histone deacetylases and the immunological network: implications in cancer and inflammation. Oncogene $\mathbf{2 9}$, 157-173 (2010).

20. Zhang, H. et al. Role of histone deacetylase expression levels and activity in the inflammatory responses of patients with chronic hepatitis B. Mol. Med. Rep. 15, 2744-2752 (2017).

21. Palazon, A., Goldrath, A. W., Nizet, V. \& Johnson, R. S. HIF transcription factors, inflammation, and immunity. Immunity 41, 518-528 (2014).

22. Yan, K. L. et al. Histone deacetylase 9 deficiency protects against effector T cellmediated systemic autoimmunity. J. Biol. Chem. 286, 28833-28843 (2011).

23. Glick, D., Barth, S. \& Macleod, K. F. Autophagy: cellular and molecular mechanisms. J. Pathol. 221, 3-12 (2010).

24. Beau, I., Mehrpour, M. \& Codogno, P. Autophagosomes and human diseases. Int. J. Biochem. Cell Biol. 43, 460-464 (2011).

25. Mizushima, N., Levine, B., Cuervo, A. M. \& Klionsky, D. J. Autophagy fights disease through cellular self-digestion. Nature 451, 1069-1075 (2008).

26. Garcia-Prat, L. et al. Autophagy maintains stemness by preventing senescence. Nature 529, 37-42 (2016).

27. Jones, A. E. et al. Wnt/beta-catenin controls follistatin signalling to regulate satellite cell myogenic potential. Skelet Muscle 5, 14 (2015).

28. Suzuki, A., Pelikan, R. C. \& Iwata, J. WNT/beta-catenin signaling regulates multiple steps of myogenesis by regulating step-specific targets. Mol. Cell Biol. 35, 1763-1776 (2015).

29. Kadir, R. et al. ALFY-controlled DVL3 autophagy regulates Wnt signaling, determining human brain size. PLoS Genet. 12, e1005919 (2016).

30. Goll, D. E., Thompson, V. F., Li, H., Wei, W. \& Cong, J. The calpain system. Physiol. Rev. 83, 731-801 (2003).

31. Min, K. et al. Increased mitochondrial emission of reactive oxygen species and calpain activation are required for doxorubicin-induced cardiac and skeletal muscle myopathy. J Physiol. 593, 2017-2036 (2015).

32. Smith, I. J., Lecker, S. H. \& Hasselgren, P. O. Calpain activity and muscle wasting in sepsis. Am. J. Physiol. Endocrinol. Metab. 295, E762-E771 (2008). 
33. Yun, Z., Lin, Q. \& Giaccia, A. J. Adaptive myogenesis under hypoxia. Mol. Cell. Biol. 25, 3040-3055 (2005).

34. Lee, S. W. et al. MicroRNA-26a induced by hypoxia targets HDAC6 in myogenic differentiation of embryonic stem cells. Nucleic Acids Res. 43, 2057-2073 (2015).

35. McKinsey, T. A., Zhang, C. L., Lu, J. \& Olson, E. N. Signal-dependent nuclear export of a histone deacetylase regulates muscle differentiation. Nature $\mathbf{4 0 8}$, 106-111 (2000).

36. Mozzetta, C. et al. Fibroadipogenic progenitors mediate the ability of HDAC inhibitors to promote regeneration in dystrophic muscles of young, but not old Mdx mice. EMBO Mol. Med. 5, 626-639 (2013).

37. Saccone, V. et al. HDAC-regulated myomiRs control BAF60 variant exchange and direct the functional phenotype of fibro-adipogenic progenitors in dystrophic muscles. Gene Dev. 28, 841-857 (2014).

38. Liu, H., Qiu, H., Yang, J., Ni, J. \& Le, W. Chronic hypoxia facilitates Alzheimer's disease through demethylation of gamma-secretase by downregulating DNA methyltransferase 3b. Alzheimers Dement. 12, 130-143 (2016).

39. Liu, H. \& Le, W. Epigenetic modifications of chronic hypoxia-mediated neurodegeneration in Alzheimer's disease. Transl. Neurodegener. 3, 7 (2014).

40. Yang, Q., Sun, M., Ramchandran, R. \& Raj, J. U. IGF-1 signaling in neonatal hypoxia-induced pulmonary hypertension: role of epigenetic regulation. Vascul. Pharmacol. 73, 20-31 (2015).

41. Watson, C. J. et al. Hypoxia-induced epigenetic modifications are associated with cardiac tissue fibrosis and the development of a myofibroblast-like phenotype. Hum. Mol. Genet. 23, 2176-2188 (2014).

42. Ryter, S. W., Cloonan, S. M. \& Choi, A. M. Autophagy: a critical regulator of cellular metabolism and homeostasis. Mol. Cells 36, 7-16 (2013).

43. Bernardi, P. \& Bonaldo, P. Mitochondrial dysfunction and defective autophagy in the pathogenesis of collagen VI muscular dystrophies. Cold Spring Harb. Perspect. Biol. 5, a011387 (2013).

44. Sandri, M., Coletto, L., Grumati, P. \& Bonaldo, P. Misregulation of autophagy and protein degradation systems in myopathies and muscular dystrophies. J. Cell Sci. 126, 5325-5333 (2013).

45. Giordano, C., Lemaire, C., Li, T., Kimoff, R. J. \& Petrof, B. J. Autophagy-associated atrophy and metabolic remodeling of the mouse diaphragm after short-term intermittent hypoxia. PLOS ONE 10, e0131068 (2015).
46. Masschelein, E. et al. Acute environmental hypoxia induces LC3 lipidation in a genotype-dependent manner. FASEB J. 28, 1022-1034 (2014).

47. Rudnicki, M. A. \& Williams, B. O. Wnt signaling in bone and muscle. Bone $\mathbf{8 0}$ 60-66 (2015).

48. Huraskin, D. et al. Wnt/beta-catenin signaling via Axin2 is required for myogenesis and, together with YAP/Taz and Tead1, active in $\| \mathrm{a} / \mathrm{ll}$ x muscle fibers. Development 143, 3128-3142 (2016).

49. Abraham, S. T. A role for the Wnt3a/beta-catenin signaling pathway in the myogenic program of C2C12 cells. In Vitro Cell Dev. Biol. Anim. 52, 935-941 (2016).

50. Hutcheson, D. A., Zhao, J., Merrell, A., Haldar, M. \& Kardon, G. Embryonic and fetal limb myogenic cells are derived from developmentally distinct progenitors and have different requirements for beta-catenin. Genes Dev. 23, 997-1013 (2009).

51. Tanaka, S., Terada, K. \& Nohno, T. Canonical Wnt signaling is involved in switching from cell proliferation to myogenic differentiation of mouse myoblast cells. J. Mol. Signal. 6, 12 (2011).

52. Suzuki, A., Scruggs, A. \& Iwata, J. The temporal specific role of WNT/betacatenin signaling during myogenesis. J. Nat. Sci. 1, e143 (2015).

53. Murphy, M. M. et al. Transiently active Wnt/beta-catenin signaling is not required but must be silenced for stem cell function during muscle regeneration. Stem Cell Rep. 3, 475-488 (2014).

54. Zhai, P. \& Sadoshima, J. Glycogen synthase kinase-3beta controls autophagy during myocardial ischemia and reperfusion. Autophagy 8, 138-139 (2012).

55. Park, C. H., Lee, B. H., Ahn, S. G., Yoon, J. H. \& Oh, S. H. Serine 9 and tyrosine 216 phosphorylation of GSK-3beta differentially regulates autophagy in acquired cadmium resistance. Toxicol Sci. 135, 380-389 (2013).

56. Gavilán, E., Sánchez-Aguayo, I., Daza, P. \& Ruano, D. GSK-3beta signaling determines autophagy activation in the breast tumor cell line MCF7 and inclusion formation in the non-tumor cell line MCF10A in response to proteasome inhibition. Cell Death. Dis. 4, e572 (2013).

57. Long, Q., Li, X., He, H. \& He, D. Autophagy activation protects shock wave induced renal tubular epithelial cell apoptosis may through modulation of Akt/ GSK-3beta pathway. Int. J. Biol. Sci. 12, 1461-1471 (2016). 